\title{
Passivity of Virtual Free-Floating Dynamics Rendered on Robotic Facilities
}

\author{
Marco De Stefano $^{1}$, Jordi Artigas ${ }^{1}$, Wolfgang Rackl ${ }^{1}$ and Alin Albu-Schaeffer ${ }^{1,2}$
}

\begin{abstract}
This paper describes a control strategy to achieve high fidelity dynamics simulation rendered on admittance controlled robotic facilities. It explores the reasons for an increasing energy found in the virtual dynamics of a freefloating satellite rendered on a six degree of freedom robot, which can lead the system to become unstable and proposes a method to cope with it. The proposed method identifies the sources of intrinsic instability provoked by time delays that are found in the computational loop of the rendered dynamics and counteracts their destabilizing effects using the passivity criteria. The performance of the system and the benefits of the method are shown in simulations and are verified experimentally.
\end{abstract}

\section{INTRODUCTION}

\section{A. Motivation}

Space debris, the collection of defunct materials in the space orbits, have continually increased over recent years [1]. Commonly adopted debris mitigation techniques might be insufficient to keep the space environment safe from accidental collisions [2]. Rather than disposing malfunctional satellites, On-Orbit Servicing (OOS) systems can undertake maintenance tasks and put them back into operation. An OOS system must be able to execute several complex tasks such as grasping and stabilizing of uncooperative freefloating satellites. The system can be controlled either in semi-autonomous or teleoperated mode with high reliability levels through a free-floating servicing robot [3]. This, in turn has the potential to extend the lifetime of satellites or accomplish their safe de-orbiting. To prepare and validate the OOS technology, an on-ground test facility is required, for testing and verification of planning and control algorithms prior to the launch. To that end, some technologies such as free-fall towers, parabolic flights, air-bearing testbeds, neutral buoyancy, suspended systems and robotic simulators [4], [5] are already available. In [6] a preliminary ground verification and feasibility of OOS was presented. At DLR$\mathrm{RMC}$, an OOS simulation facility [7] has been recently developed, aimed at on-ground testing and verification of robotic components for an OOS mission. This facility is composed of three robots: a six degrees of freedom (DoF) industrial robot, that simulates the free-floating dynamics of a target satellite (see Fig. 1); and a second one of the same type equipped with a 7 DoF Light Weight Robot (LWR) mounted on its end-effector, that simulates a servicer

\footnotetext{
${ }^{1}$ The authors are with the Institute of Robotics and Mechatronics (RMC), German Aerospace Center (DLR), 82234 Weßling, Germany, email: name.lastname@dlr.de.

2 The author is with the Technical University Munich (TUM), Department of Computer Science.
}

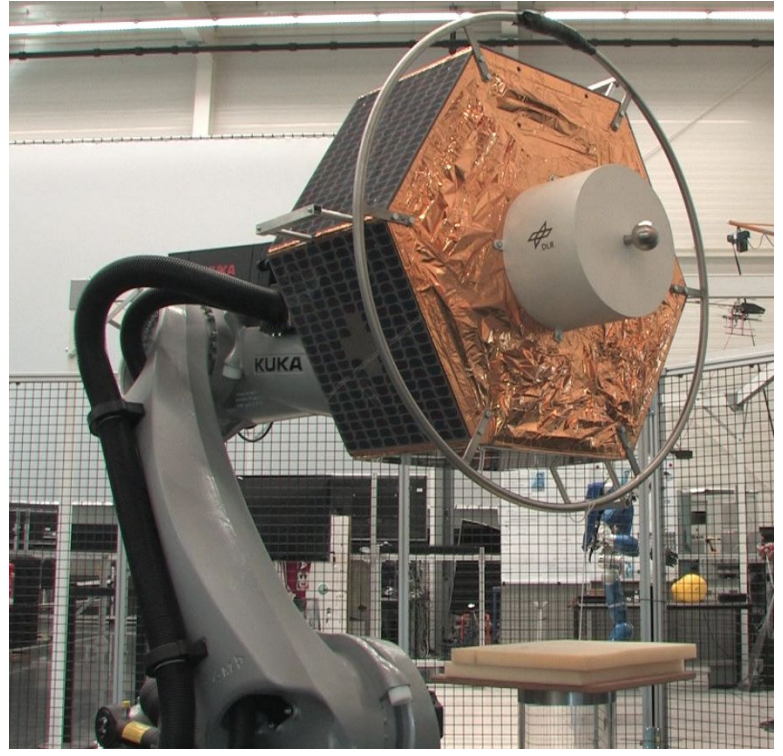

Fig. 1. Free floating satellite simulator at DLR - RMC

satellite, resulting in a macro-micro configuration with $6+7$ DoF.

A crucial question to be answered is how well the dynamics of a free-floating satellite can be rendered using robot based simulation facilities. In order to make the simulated dynamics reactive to physical interactions, force-torque sensors are used on the robot end-effectors to capture external forces. Thus, the Newton-Euler equations can be integrated in an admittance like configuration, where the motions of the simulated free-floating satellite are related to the sensed forces and torques. Nevertheless, the formality given by this classical mechanics relationship is partly broken due to the discrete computer-based implementation. Discretization, quantization and transmission delays are factors that distort the simulation. This results in additional undesired dynamics that can compromise the stability of the system. In particular, time delay is a well known factor that can disrupt system stability.

\section{B. Related Works}

A similar phenomenon has been investigated in the field of haptics and teleoperation ${ }^{1}$ in an impedance like configuration [8], where time delay and zero-order-hold are shown to be energy generating, resulting in an active system causing

\footnotetext{
${ }^{1}$ The haptic device takes computed forces as input and outputs its current position
} 
instability. In [9] the contact dynamics of a hardware-in-theloop facility for on-orbit docking is analyzed; the contact is assumed to be sufficiently long such that the time delay does not play any role. In [10] a minimum virtual mass passivity value based on Colgate's [10] passivity condition is found for a haptic device in an impedance like configuration. In contrast to the previous works, [11] and [12] consider passivity in the time domain, the latter making use of the Time Domain Passivity Control Approach (TDPA) [13] for multi DoF in impedance configurations.

Complex discrete systems often include interconnected algorithms that run in diffrent CPUs. It is well known that lowlevel control is very sensitive to time delays. The approach pursued in this paper is developed in the electrical domain. As it will be seen, the electrical representation unveils active two-port networks that are created by the time delays found in the (distributed) control loop. These networks, called Time Delay Power Networks (TDPN) [14], are responsible for transferring energy between algorithms that run on different CPUs. The main benefit of using the TDPN modeling is that they allow to identify and isolate energy leaks, that is, undesired sources of energy that can lead the system to become unstable. Once the TPDN are identified, their energy can be controlled in order to counteract the damaging effects on the system stability. The Time Domain Passivity Control strategy is here applied to that end for two-port networks in an admittance-like configuration, where forces are fed into the controller and desired position signals are commanded. To the best of the authors' knowledge, none of the previous studies address the time domain passivity treatment of an admittance-like dynamics simulation in order to explicitly counteract the effects of time delay. As will be seen, these effects become apparent in contact situations when the rendered mass collides with physical objects. The approach has been tested in a single DoF system and simulated for a six DoF free-floating rigid body that represents a satellite.

The paper is organized as follows: the problem statement is discussed in section II. In section III, a network model, which isolates the instability source is proposed as a method to identify the energy leaks. The control scheme, based on the passivisation of this network, is shown in section IV. Moreover the efficiency of the method is proven with simulations in section $\mathrm{V}$ and experimentally on a 1 DoF setup in section VI.

\section{Problem Statement}

Current robotic facilities to simulate free-floating dynamics in six DoF use industrial robots in order to fulfill workspace and dynamic related requirements [5], [7] . Usually these robots can be controlled only by means of position commands. This poses an added difficulty in the rendering and control of a virtual mass due to the absence of a torque interface. In turn, this imposes an admittance causality on the passivity controller, that is, the modified variable can only be position (as opposed to the impedance type, which acts on force signal modifications). The general system studied in this paper considers a 6 DoF industrial robot manipulator with a force-torque sensor placed at its end-effector to capture the physical interaction. The freefloating dynamics of a rigid body (6 DoF), based on the Newton-Euler equations [15], is used to compute the desired position command to the industrial robot as:

$$
\begin{gathered}
\mathbf{v}(t)=\int_{0}^{t} \frac{\boldsymbol{F}_{M}(t)}{M} d t \\
\boldsymbol{\omega}(t)=\int_{0}^{t} \boldsymbol{I}^{-1}\left(\boldsymbol{\tau}_{\boldsymbol{M}}(t)-\boldsymbol{\omega}(t) \times \boldsymbol{I} \boldsymbol{\omega}(t)\right) d t .
\end{gathered}
$$

where $\mathbf{v}(t) \in \mathbb{R}^{3 \times 1}$ is the linear velocity of the Center of Mass (CoM) and $\boldsymbol{\omega}(t) \in \mathbb{R}^{3 \times 1}$ is the CoM angular velocity. $\mathbf{F}_{M}(t) \in \mathbb{R}^{3 \times 1}$ are the forces and $\boldsymbol{\tau}_{M}(t) \in \mathbb{R}^{3 \times 1}$ are the torques applied on the CoM of the satellite frame where the gravity components are compensated in the model. The mass and inertial characteristics are $M \in \mathbb{R}$ and $\mathbf{I} \in \mathbb{R}^{3 \times 3}$ which represent the simulated virtual mass. Without loss of generality, by integrating (1) and (2) and using the EulerRodrigues formulation [16], a desired transformation matrix $\mathbf{T}_{\text {sat }} \in \mathbb{R}^{4 \times 4}$ is defined as input to the industrial robot using the inverse kinematics. Accordingly, the commands to the robot are desired positions in the Cartesian space, which in turn render the satellite dynamics. Fig. 2 shows the interface architecture. The facility consists of a KR120 industrial robot [7] provided with a 6 DoF Force-Torque Sensor (FTS) at its end effector. The forces and torques are measured with the sensor and are used for the computation of the simulated dynamics. Eq. (1)-(2) are thus implemented in a real-time computer with a sampling time of $1 \mathrm{~ms}$. Furthermore, the commands are sent to the Robot Control

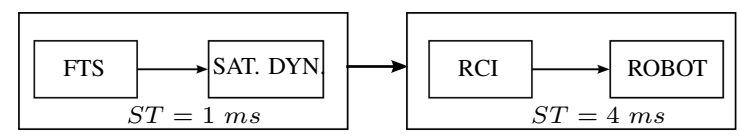

Fig. 2. Interface architecture

Interface $(R C I)$ at a rate of $4 \mathrm{~ms}$. The total round trip delay between the commanded position and the measured position, was measured to be $16 \mathrm{~ms}$. Zero Order Hold ( $\mathrm{ZOH})$, time discretization and time delay are intrinsic in the control loop and they are sources of energy. In discrete time this energy is calculated by:

$$
\begin{array}{r}
E(n)=E(0)+\sum_{k=0}^{n} \tau_{M}(k) \omega(k-\mu) \Delta T+ \\
\sum_{k=0}^{n} F_{M}(k) v(k-\mu) \Delta T,
\end{array}
$$

where $\mu$ is the number of discrete-time steps of sampling time $\Delta T$ equivalent to a continuous-time time delay $T_{d}$, i.e, $T_{d}=\mu \Delta T$ with $\mu \in \mathbb{N}$.

The energy associated with the simulated dynamics, will be also affected by the factors mentioned earlier. In order to show this phenomenon, we assume a body with a defined virtual inertia. Its CoM is free to rotate around its rotational 
axis in 1-DoF between two rigid walls. According to Euler laws of motion, the body should keep rotating with constant velocity. Fig. 3 shows the energy $E_{i}$ in the ideal case (dashed line) and the energy $E_{T D}$ considering the time delay effects. A delay of $10 \mathrm{~ms}$ is considered here. The time delay introduces negative energy (by means activity) in the system. As can be seen in the bottom plot of Fig. 3, the desired
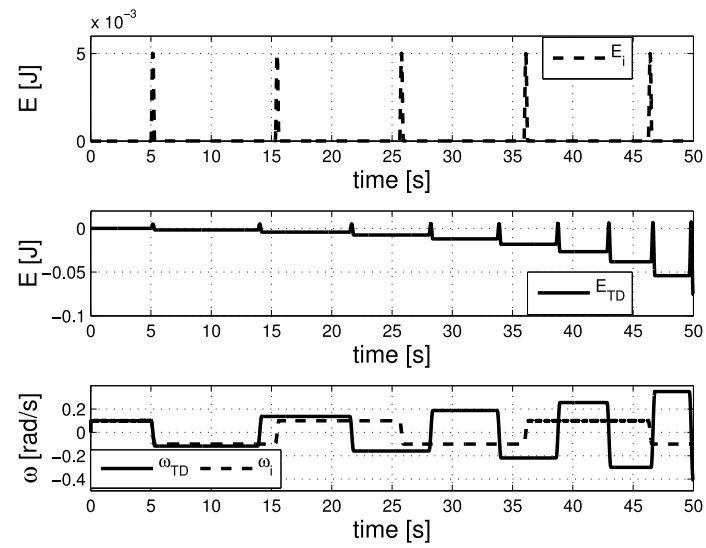

Fig. 3. Simulated virtual mass bouncing between two walls: $E_{i}$ : ideal energy, $E_{T D}$ : energy considering the $T_{d}$ effect, $\omega$ : angular velocity

dynamics is affected. The time delay in the network causes a divergence of the angular velocity $\omega_{T D}$ with respect to the ideal velocity $\omega_{i}$. For this reason, the system has an active behaviour. The passivity of a network can be checked using the quadratic norm of its scattering operator $S$ with the condition $|S|^{2} \leq 1$. It can be proved that, if a network contains a delay, no matter how small, the passivity condition is not fulfilled [17]. Brown and Colgate proposed in [10] a minimun mass which can be simulated passively. It is proven that no explicit passive numerical algorithm exists, unless the virtual mass is greater than this minimum value. As a matter of fact, the described satellite simulator works according to the Colgate criterion. It is not the aim of this article to explore the minimum allowed mass for stability, but rather to identify energy leaks of the system, like time delay, which can destabilize the system. The benefit of using TDPA is that it can be applied without detailed models of the system, including the robot dynamics. Moreover, using the network representation, the passivity of a network can be analyzed by checking the power contribution of each network. By definition in [13], the one-port network $N$ with initial energy $E(0)$ at time $t=0$ is continuous time passive if and only if:

$$
E(t)=E(0)+\int_{0}^{t} F(t) v(t) d t \geq 0, \quad \forall t>0
$$

with Force $F(t) \in \mathbb{R}$ and velocity $v(t) \in \mathbb{R}$. TDPA introduces the Passivity Observer (PO), and the Passivity Controller (PC) able to create a passive network.

\section{PRoposed Method}

The robot dynamics used in the proposed method is described in Cartesian space. The manipulator equation is defined as:

$$
\Lambda\left(x_{e}\right) \ddot{x}_{e}+\eta\left(x_{e}, \dot{x}_{e}\right) \dot{x}_{e}+g\left(x_{e}\right)=\Gamma,
$$

where $\boldsymbol{\Lambda} \in \mathbb{R}^{6 \times 6}$ is the cartesian matrix, $\boldsymbol{\eta} \in \mathbb{R}^{6 \times 6}$ is the coriolis cartesian matrix and $\boldsymbol{g} \in \mathbb{R}^{6 \times 1}$ is the gravity vector. $\Gamma \in \mathbb{R}^{6 \times 1}$ represents the contribution of the end-effector forces $\boldsymbol{F}_{\boldsymbol{C}}$, due to the joint actuator, and $\boldsymbol{F}$ represents the forces due to the contact with the environment. $\boldsymbol{F}_{\boldsymbol{C}}$ can be written as:

$$
F_{C}=F+\Gamma .
$$

The cartesian terms are expressed as folllow:

$$
\begin{array}{r}
\Lambda\left(x_{e}\right)=J^{-T} M J^{-1} \\
\eta\left(x_{e}, \dot{x}_{e}\right) \dot{x}_{e}=J^{-T} C \dot{q}-\Lambda \dot{j} \dot{q} \\
g\left(x_{e}\right)=J^{-T} G .
\end{array}
$$

where $M, C, G$ are the inertia matrix, the coriolis matrix and the gravity vector expressed in joints space. We assume that the Jacobian $\boldsymbol{J} \in \mathbb{R}^{6 \times 6}$ has full row rank and singularities are not taken into account.

In the simulation model, the external forces $\mathrm{F}$ which simulate the sensor are modeled as a virtual environment via a discrete-time spring damper with high stiffness given by the transfer function:

$$
H(z)=K_{\text {wall }}+D_{\text {wall }} \frac{z-1}{\Delta T z},
$$

where $K_{\text {wall }}$ is a stiffness gain and $D_{\text {wall }}$ is a damping gain. In order to analyze the stability of the system, the following simplifications and assumptions are used:

- The controller of the robot is known.

- Only the effects of the time delay are considered.

The modeling process is divided into two steps: first, the system is represented as a block diagram. The block diagram is a straightforward representation of the system as an interconnection of transfer functions and feedbacks. However, if energy considerations are to be taken into account, this representation might not be well suited. To that end, we propose a second step using the mechanical-electrical analogies, which maps the model into the electrical domain. As it will be seen, this facilitates the passivity analysis and helps to identify energy leaks, that is, undesired sources of energy that appear due to the delay in the system.

\section{A. Block diagram}

Fig. 4 shows the block diagram of the considered system. The robot is represented as an admittance $R$ (that generates a velocity $\left.v_{i s}\right), C$ is the controller and we assume that both are known. $E$ is the virtual environment modeled in the simulation as (10). During the experiments, the reaction of the environment will be measured by a forces and torques sensor. For the sake of simplicity, the overall time delay is concentrated at a single location in the model $(T D)$. In the 
proposed scheme, stability is dependent on the amount of delay but not on its location. ${ }^{2}$ The satellite dynamics is represented by a single block, whose output is the velocity vector $v_{1}(t)$ and inputs are the forces and torques $F$, measured by

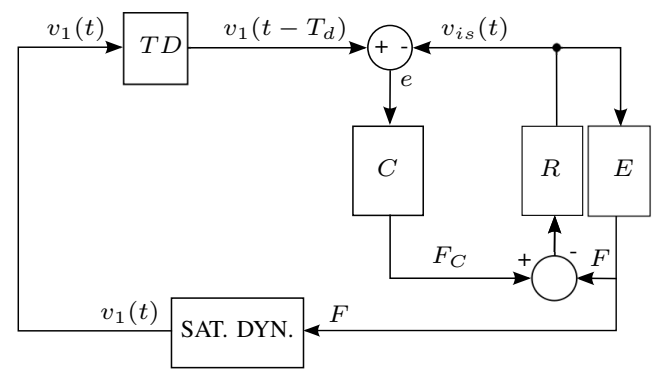

Fig. 4. Model of the problem statement

the sensor. $v_{1}(t)$ is a $6 \mathrm{x} 1$ vector composed of $v(t)$ from (1) and $\omega(t)$ from (2), $F$ is the $6 \times 1$ forces/torques vector:

$$
v_{1}(t)=[v(t), \omega(t)]^{T} ; F(t)=\left[F_{M}(t), \tau_{M}(t)\right]^{T} .
$$

\section{B. Electrical and Network representation}

Analogies are useful since they allow to analyze a domain by means of elements and laws, that belong to another. In this context, it is convenient to analyze the system as Hamiltonian ports [18] or, as considered in this paper, in electrical domain. The conventional mechanical-electrical analogy, namely velocity-current analogy, maps forces into voltages and velocities into currents. The model represented in the electrical domain is shown in Fig. 5. As it can be seen, the electrical scheme unveils the power conjugated pairs that describe each network port in the system. The forces and

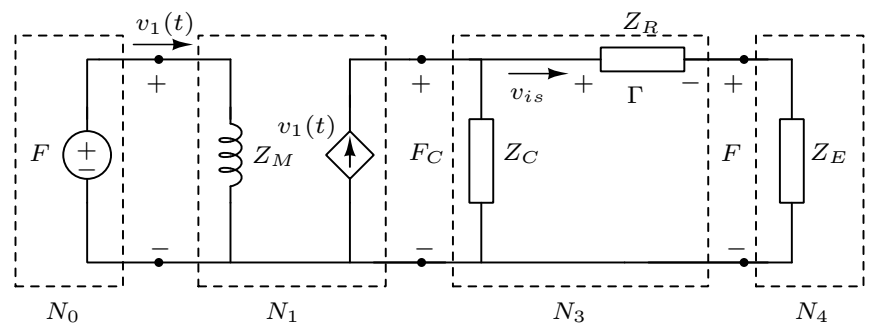

Fig. 5. Electrical analog of the system in Fig. 4

torques coming from the sensor are modeled as an ideal force generator $F$ that acts on the impedance $Z_{M}$, which represents the virtual mass and its analog is an inductance. Through a dependent current source, the velocity through the mass is fed to the controller $Z_{C}$, which, in turn, moves the robot, represented by a general impedance, $Z_{R}$. The environment is represented by an impedance $Z_{E}$. Furthermore, Fig. 5 shows one and two-port networks of the system where $N_{0}$ is a one-port network containing the sensor and $N_{1}$ is a two-port transmission network; Fig. 5 shows the ideal case, where the velocity of the virtual mass is commanded to the robot (through the controller). $N_{3}$ is a network containing the robot

\footnotetext{
${ }^{2}$ As long as the delay location is anywhere in the main loop.
}

and a position controller. The force across the controller (and the robot) is given by:

$$
\begin{aligned}
F_{C} & =v_{1}(t) \frac{Z c(t) Z_{R E}(t)}{Z c(t)+Z_{R E}(t)} \\
Z_{R E} & =Z_{R}(t)+Z_{E}(t)
\end{aligned}
$$

$Z_{R E}$ being the equivalent serial impedance of the robot and the environment.

In the proposed electrical scheme in Fig. 6, it is assumed that the time delay is located between the networks $N_{1}$ and $N_{3}$ and it produces a delayed velocity $v_{1}\left(t-T_{d}\right) . N_{2}$, composed of $Z_{P C}$, is a time varying damper, which, as will be shown in the upcoming section, will ensure the stability of the system by dissipating the active energy introduced due to the time delay. One of the main benefits of the network representation is that it allows to analyze the passivity of the system. In Fig. 6, the network $N_{T}$ is a two-port network, which contains a pure time delay. It can be proven, that such a network cannot be guaranteed to be passive due to the phase lag [17]. Thus, $N_{T}$ represents an active network, with input $v_{1}(t)$ and

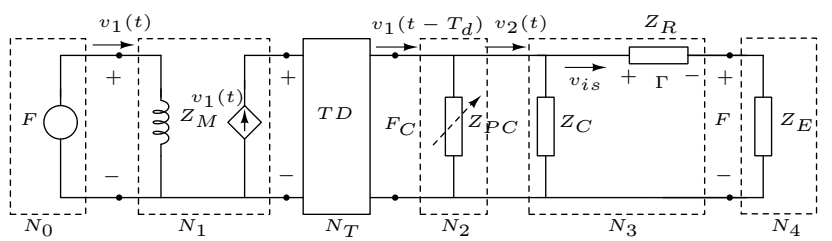

Fig. 6. Proposed system in the electrical / network domain with the PC

output $v_{1}\left(t-T_{d}\right)$, and cross voltage, i.e. $F_{C}$. We propose the electrical scheme in Fig. 6 as a new approach for a robotic closed loop system that contains internal communication delays. Based on this abstraction, passivity as a stabilization tool is applied to ensure stable performance while conserving the desired simulated momentum of the virtual mass.

\section{6 DOF PASSIVITY APPLIED TO THE SATELLITE DYNAMICS WITH TIME DELAY}

As previously stated, passivity is a sufficient condition for stability and can be applied to linear and nonlinear systems. The TDPA strategy is used to make the discrete virtual mass dynamics passive. The effects of time delay are also considered and the system, thus, is rendered passive. Furthermore, passivity of individual network elements of a cascaded network ensures overall system passivity and in turn, stability. The Passivity Observer (PO) is here defined by looking at the network $N_{T}$. The PO observes the energy of the network and the PC acts as a variable damper modulated according to the amount of active energy observed by the PO.

\section{A. Passivity Observer (PO)}

The network $N_{T}$ shown in Fig. 6 describes the active network to be passivated. $N_{T}$ is a two-port network where the net energy, which is the difference between the input and 
output energies, can be calculated as:

$$
\begin{aligned}
E_{o b s, i}(n)=\sum_{k=0}^{n} F_{C, i}(k) v_{1, i}(k) \Delta T & \\
& -\sum_{k=0}^{n} F_{C, i}(k) v_{1, i}(k-\mu) \Delta T,
\end{aligned}
$$

where the time delay is taken into account and $v_{1}(k)$ is the velocity vector of the body, $F_{C}(k)$ refers to the controller forces and torques and $v_{1}(k-\mu)$ is the delayed velocity. To observe the energy, we handle passivity for each degree of freedom, $i$, independently.

\section{B. Passivity Controller (PC)}

The Passivity Control is based on an admittance behavior with a variable inverse damping factor $\beta$, which adapts as a function of the observed energy flow (12). In the admittance configuration, the velocity is modified to produce the dissipation. The modified velocity $v_{2}$, which is the output of the PC, is described as follows:

$v_{2, i}(n)= \begin{cases}v_{1, i}(n-\mu) & E_{o b s, i} \geq 0 \\ v_{1, i}(n-\mu)-\beta_{i}(n-1) F_{C, i}(n) & E_{o b s, i}<0\end{cases}$

where $v_{1}(n-\mu)$ is the delayed velocity signal coming from the simulated dynamics, $\beta(n-1)$ is the time varying damping factor which alters the velocity by a quantity $\Delta v(n)$, given by:

$$
\Delta v_{i}(n)=-\beta_{i}(n-1) F_{C, i}(n)
$$

In order to define $\beta$, we need to combine the energy observed in the network $N_{T}$ and the energy of the passivity controller. It results into the following expression for the energy flow:

$$
\begin{gathered}
E_{o b s, i}(n)=E_{o b s, i}(n-1)+E_{p c, i}(n)= \\
E_{o b s, i}(n-1)+\sum_{k=0}^{n} F_{C, i}^{2}(k) \beta_{i}(k-1) \Delta T,
\end{gathered}
$$

where $E_{p c, i}$ is the dissipated energy by the damper $\beta$. The passivity condition is then applied as: $E_{o b s, i}+E_{p c, i} \geq 0$, which leads to the following damping coefficient:

$$
\beta_{i}(n)= \begin{cases}-\frac{E_{o b s, i}(n)}{F_{C, i}^{2}(n) \Delta T} & E_{o b s, i}<0 \\ 0 & E_{o b s, i} \geq 0\end{cases}
$$

Thus, the network created by $N_{T}$ along with $N_{2}$, i.e., the passivity controller, is passive. The problem is solved using the proposed method to passivate the two-port network. The modified problem statement is illustrated in Fig. 7. $R$ is the robot and $C$ is the internal controller of the robot. Moreover, the $P C$ controller block and the $P O$ observer block are added in the loop to modulate the energy leaks in the channel due to the time delay $T_{d}$.

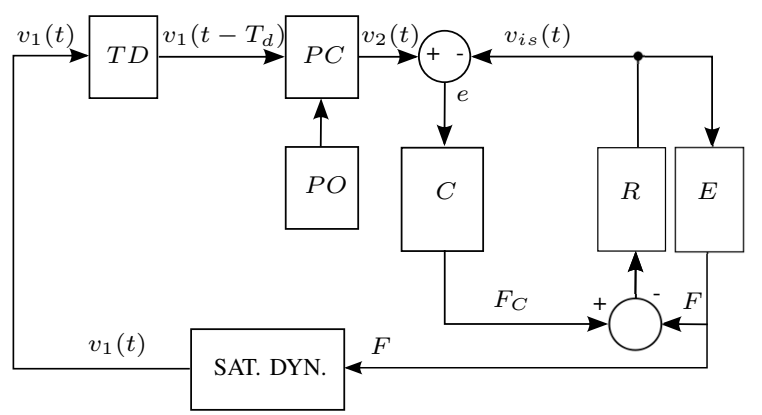

Fig. 7. Problem statement with PC

\section{Simulation}

External disturbances due to collision increase the source of activity. In the first simulation, an initial angular velocity is given for the satellite in order to simulate a spin around its inertial axis. Virtual rotational springs with high stiffness are modeled in order to simulate the FTS sensors and the body is spinning between them. In the case of an ideal impact, the rotational velocity before $\left(\omega_{1}\right)$ and after $\left(\omega_{2}\right)$ the impact are the same according to the principle of conservation of energy. ${ }^{3}$ The data in Fig. 8 are simulated for a body with $\operatorname{diag}(I)=0.9 \mathrm{Kgm}^{2}$ and with a sampling time of $1 \mathrm{~ms}$. The solid line displays the simulated ideal case of the body bouncing between the two walls without any time delay. The initial angular velocity is $0.1 \mathrm{rad} / \mathrm{s}$ on the $z-$ axis. After each impact, the angular velocity $\omega$ remains constant

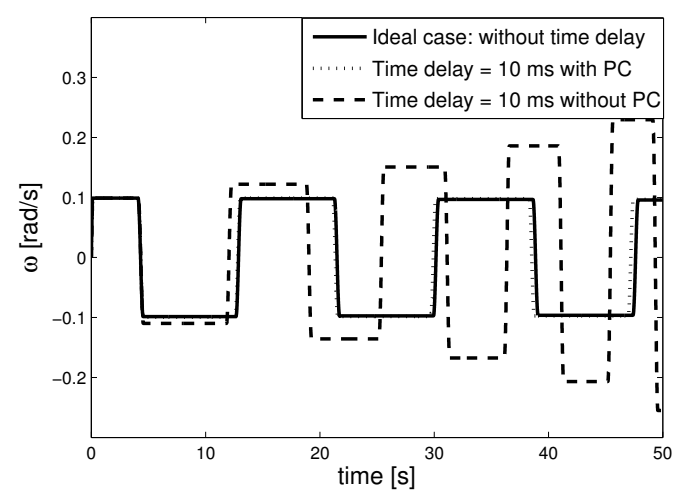

Fig. 8. Angular velocity of the simulated Inertia without time delay for the ideal case (solid line), with $10 \mathrm{~ms}$ time delay and PC enabled (dotted line), with $10 \mathrm{~ms}$ time delay and PC disabled (dashed line).

in magnitude as expected from the principle of conservation of energy. As illustrated in Fig. 8, if a time delay of $10 \mathrm{~ms}$ is present in the loop without PC, the angular velocity increases (dashed line) after each impact. The time delay results in an active behavior in the system. The dotted line in Fig. 8 illustrates the effectiveness of the proposed method, where for the same time delay, the angular velocity is maintained very close to the ideal case by the PC. The mentioned problem described in section II by Fig. 3 is here solved using

\footnotetext{
${ }^{3}$ For an elastic impact the rotational velocity after each impact will decrease due to energy dissipation effects
} 

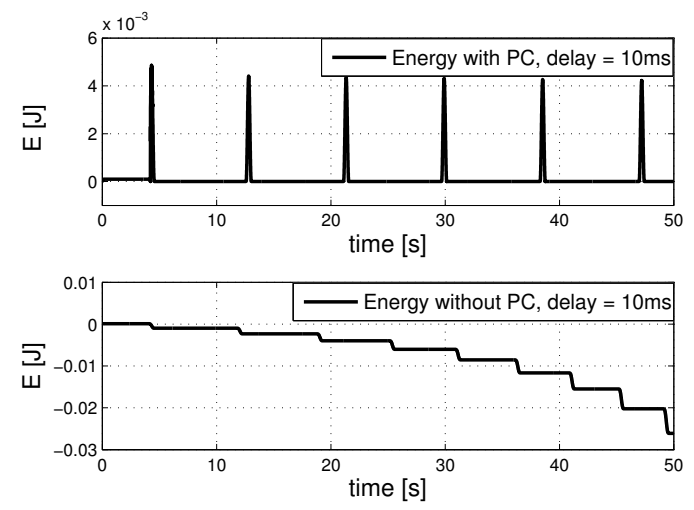

Fig. 9. Energy of the network with $10 \mathrm{~ms}$ time delay: with PC (top) and without PC (bottom)

the described approach. Moreover, Fig. 9 shows the energy behavior of the network. The positive trend of the energy on the top plot shows a passive behavior of the network by virtue of the PC. On the other hand, the bottom plot clearly shows the active behavior of the network without PC, leading to an increase of the angular velocity. The growing negative value in the energy shows that energy is injected into the system after each contact. The second simulation shows the interaction of a simulated mass of $50 \mathrm{Kg}$ with an initial velocity $v_{1}=[0.1,0.05,0.1] \mathrm{m} / \mathrm{s}$ and a time delay of $10 \mathrm{~ms}$. The virtual wall has been modeled as high stiffness springs to simulate a collision. The velocity in Fig. 10
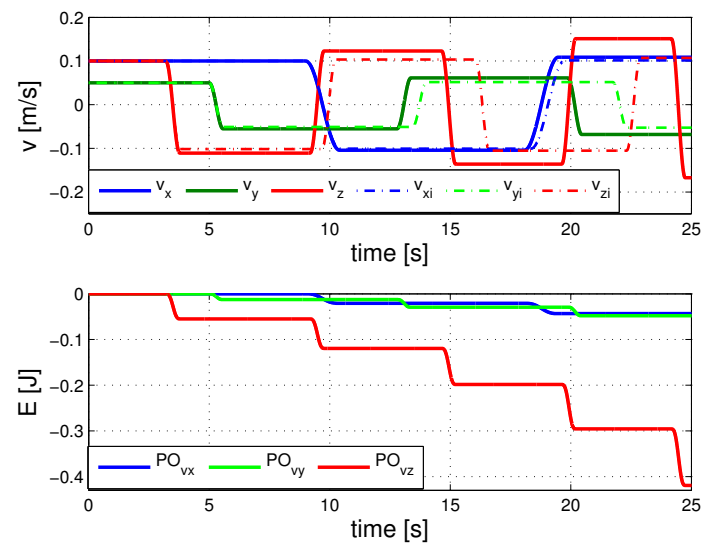

Fig. 10. Velocity of the simulated mass in the ideal case (dashed line) and without PC (solid line). At the bottom, the Passivity Observer without PC

shows the unstable behavior given by the time delay. The dashed line is the ideal motion of the satellite obtained in the ideal case (without time delay). At each impact, the velocity increases along all the components. Further, active energy is introduced as the Passivity Observer in Fig. 10 bottom shows. By applying the method (see Fig. 11), the velocity of the simulated mass follows the one given by the ideal motion (dashed line). The network is then passive as Fig. 11 shows. As it can be seen, the simulations prove the validity of the proposed approach.
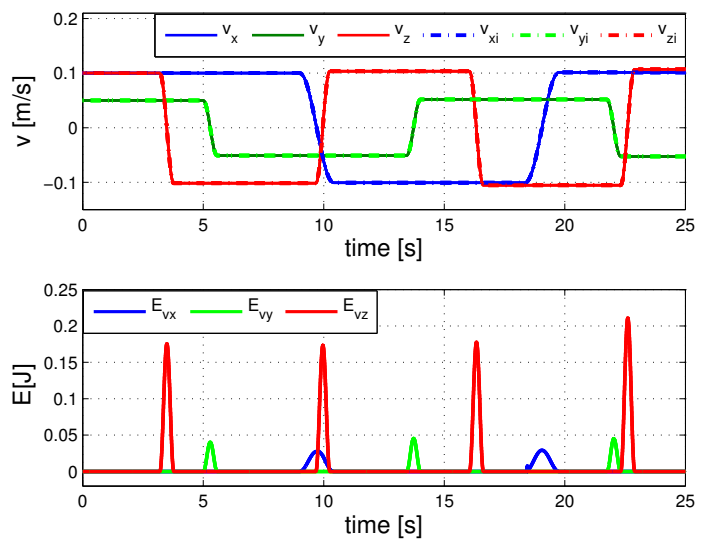

Fig. 11. Velocity of the simulated mass in the ideal case (dashed line) and with PC (solid line). At the bottom, the Passivity Observer with PC

\section{EXPERIMENTS}

Due to the highly unstable behavior of the robots without the use of passivity control, preliminary experiments are carried out on a 1 DoF SensorDrive set-up (see Fig. 12 a.) equipped with a torque sensor and a rigid bar attached to it. The bar is a mechanical interface which makes contacts
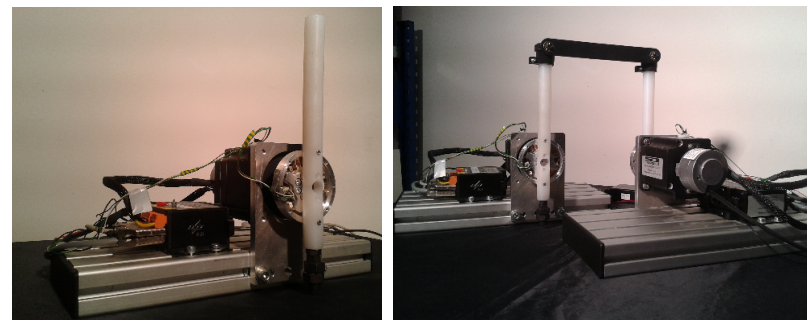

Fig. 12. a. One DoF set-up

b. Two bodies connected via a rigid link

with the rigid walls on both sides. It can be seen as a 1 DoF spinning satellite where the inertial matrix in (2) is a scalar, the vector in (11) and $F_{C}$ are composed of only one component. The intrinsic delay of the system was measured to be $2 \mathrm{~ms}$. The embedded SIMULINK model runs on a $Q N X$ real-time operating system at $1 \mathrm{~ms}$ sampling rate. Furthermore the internal controller was parametrized for a maximum performance assuming an ideal case, i.e. with high stiffness and nearly-null damping. Two experiments are shown here to prove the validity of the method. The initial angular velocity of the system is $\omega_{\text {init }}=0.473 \mathrm{rad} / \mathrm{s}$ and the time delay is $10 \mathrm{~ms}$. The virtual inertia to be simulated is $I=0.009 \mathrm{Kgm}^{2}$. The choice of a small value for inertia is made to minimize the contact time which allows us to see the influence of the time delay. Fig. 13 shows the experimental results for the system without the PC. The bottom plot clearly shows that after each contact with the wall, the angular velocity increases as expected from the simulation and reaches the value of $\omega=1.255 \mathrm{rad} / \mathrm{s}$ after $t=30 \mathrm{~s}$. Fig. 14 shows the results of the experiment under the same conditions, but with the PC enabled. It can be seen that 

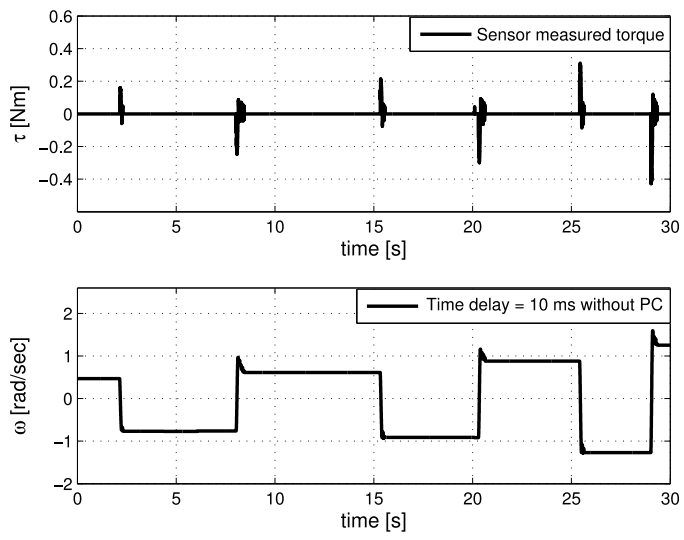

Fig. 13. Experimental results showing an active behavior of the simulated virtual mass without $\mathrm{PC}, T_{d}=10 \mathrm{~ms}$

through out the duration of the experiment, the magnitude of the angular velocity remains constant. The generated energy due to the time delay is now damped making the network (and therefore the system) passive. The experimental proof
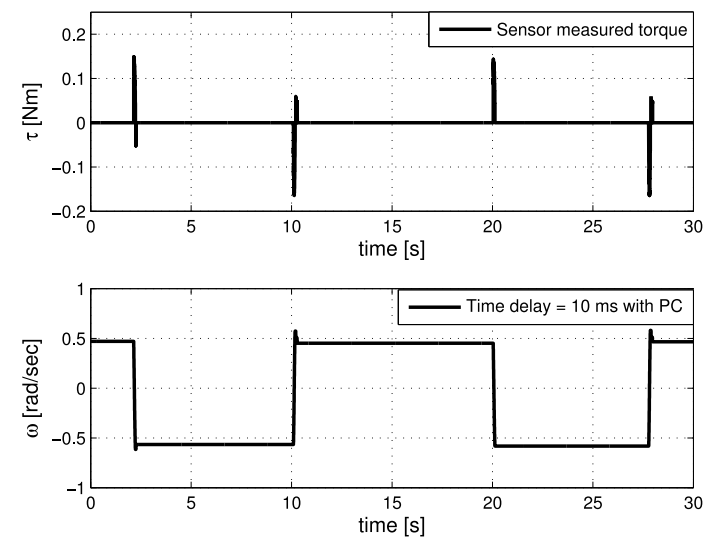

Fig. 14. Experimental results showing a passive behavior of the simulated virtual mass applying the $\mathrm{PC}, T_{d}=10 \mathrm{~ms}$

of the concept can be seen in Fig. 15 in which the top plot shows the energy behavior of the network and the bottom plot shows the velocity correction made by the PC in (13). The energy of the network is always positive due to the PC. Furthermore, instability features are not detected proving the validity of the proposed method. The method is also extended and experimentally tested for two virtual bodies connected using a rigid link, see Fig. 12b. This can be seen as a 1 DoF analogy to an on-ground OOS scenario where a client satellite is rigidly grasped by a robotic arm mounted on a free-floating servicer satellite. It is verified that the local time delays have strong influence on the performance of the coupled system. Only by applying the proposed method, it was possible to avoid instability features. The behavior and the performance of the single DoF and the coupled system for both cases (with and without PC) can be seen in the
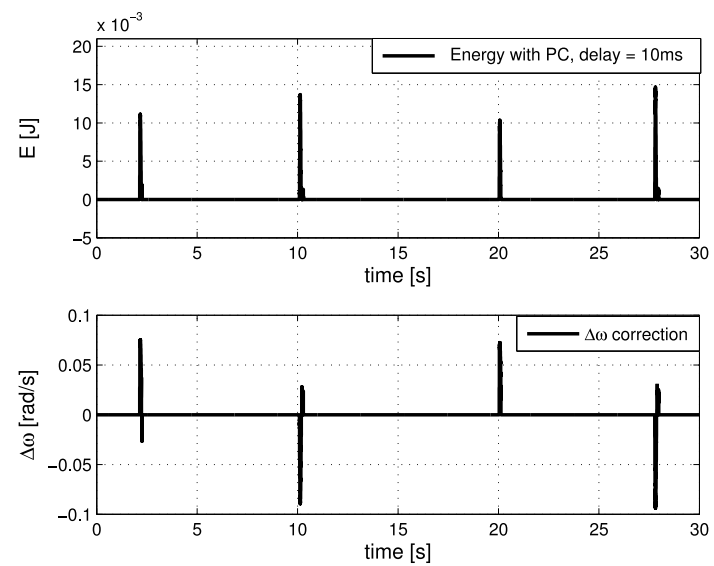

Fig. 15. Experimental proof applying the PC: Energy (top), admittance correction (bottom)

accompanying video.

\section{CONCLUSION AND FUtURE WORKS}

This work has presented a method for mitigating the effects of discretization dynamics that distort the simulation of a virtual dynamics which can potentially lead the system to become unstable. In particular, the time delay in the control loop, needed to simulate the virtual dynamics is found to change the desired properties and it can destabilize the system. The reason for that is the generation of virtual energy induced by the delay. An admittance control approach has been used to lead the rendered dynamics by a robot close to the simulated dynamics. The approach has been tested in single DoF set-up and simulated for a six DoF free-floating rigid body, that is, the satellite. The treatment of the problem in the electrical domain and its network abstraction allow to analyze the system in terms of energy. The Time Domain Passivity Control provides in this context a powerful tool that allows to passivate a two-port network which is identified to be active due to the time delay. Next to the mathematical development of the proposed method, empirical stability tests illustrated the effectiveness of the approach: a freefloating bouncing mass against two walls; and a rigid linkage connecting two free-floating masses. The results of both tests are presented also in the accompanying video. The approach is aimed at generality so that discretization and quantization effects can also be treated in a similar manner and this remains an issue for future work.

\section{ACKNOWLEDGMENT}

The authors would like to gratefully acknowledge the valuable comments and suggestions from Ribin Balachandran during the writing of the paper.

\section{REFERENCES}

[1] N. L. Liou, J.-C.; Johnson, "A sensitivity study of the effectiveness of active debris removal in leo," in Acta Astronautica, vol. 64, no. 2-3, 2009, pp. $236-243$.

[2] N. L. N. H. Liou, J.-C.; Johnson, "Controlling the growth of future leo debris populations with active debris removal," in Acta Astronautica, vol. 66, no. 5-6, 2010, pp. 648-653. 
[3] K. Landzettel, C. Preusche, A. Albu-Schaffer, D. Reintsema, D. Rebele, and G. Hirzinger, "Robotic on-orbit servicing - dlr's experience and perspective," in Intelligent Robots and Systems, 2006 IEEE/RSJ International Conference on, Oct 2006, pp. 4587-4594.

[4] J. L. Schwartz, M. A. Peck, and C. D. Hall, "Historical review of airbearing spacecraft simulators," in Journal of Guidance, Control and Dynamics, 2003, pp. AAS 03-125.

[5] F. Sellmaier, T. Boge, J. Spurmann, S. Gully, T. Rupp, and F. Huber, "On-orbit servicing missions: Challenges and solutions for spacecraft operations," in American Institute of Aeronautics and Astronautics, Inc.. SpaceOps 2010 Conference, 25,Huntsville, Alabama, USA, 2010.

[6] E. Stoll, U. Walter, J. Artigas, C. Preusche, P. Kremer, G. Hirzinger, J. Letschnik, and H. Pongrac, "Ground verification of the feasibility of telepresent on-orbit servicing," in Journal of Field Robotics, vol. 26, 2009, pp. 287-307.

[7] J. Artigas, M. De Stefano, W. Rackl, B. Brunner, W. Bertleff, R. Burger, R. Lampariello, O. Porges, A. Giordano, C. Borst, and A. Albu-Schaeffer, "The oos-sim: an on-gound simulation facility for on-orbit servicing robotic operations," in Proceedings to Robotics and Automation (ICRA), 2015 IEEE International Conference.

[8] N. Diolaiti, G. Niemeyer, F. Barbagli, and J. Salisbury, "Stability of haptic rendering: Discretization, quantization, time delay, and coulomb effects," Robotics, IEEE Transactions on, vol. 22, no. 2, pp. 256-268, April 2006.

[9] M. Zebenay, R. Lampariello, T. Boge, and D. Choukroun, "A new contact dynamics model tool for hardware-in-the-loop docking simulation," in 11th i-SAIRAS 2012, Turin, Italy, 2012.

[10] J. Brown and J. E. Colgate, "Minimum mass for haptic display simulation," in Dynamic System and Control Division ASME, 1998 pp. 249-256.

[11] K. Kanaoka and T. Yoshikawa, "Passivity monitor and software limiter which guarantee asymptotic stability of robot control systems,' in Robotics and Automation, 2003. Proceedings. ICRA 'O3. IEEE International Conference on, vol. 3, Sept 2003, pp. 4366-4373.

[12] K. Hertkorn, T. Hulin, P. Kremer, C. Preusche, and G. Hirzinger, "Time domain passivity control for multi-degree of freedom haptic devices with time delay," in Robotics and Automation (ICRA), 2010 IEEE International Conference on, May 2010, pp. 1313-1319.

[13] B. Hannaford and J.-H. Ryu, "Time domain passivity control of haptic interfaces," in Robotics and Automation ICRA, 2001 IEEE International Conference on, vol. 2, 2001, pp. 1863-1869.

[14] J. Artigas, J.-H. Ryu, C. Preusche, and G. Hirzinger, "Network representation and passivity of delayed teleoperation systems," in Intelligent Robots and Systems (IROS), 2011 IEEE/RSJ International Conference on, Sept 2011, pp. 177-183.

[15] P. C. Hughes, Ed., Spacecraft Attitude Dynamics. Dover Publications, Inc., Mineola, New York, 2004.

[16] B. Siciliano and O. Khatib, Eds., Springer Handbook of Robotics. Springer, 2008.

[17] R. Anderson and M. Spong, "Bilateral control of teleoperators with time delay," Automatic Control, IEEE Transactions on, vol. 34, no. 5 , pp. 494-501, May 1989.

[18] C. Secchi, S. Stramigoli, and C.Fantuzzi, Eds., Control of interactive robotic interfaces A port-Hamiltonian approach. ser. Springer Tracks in advanced robotics. New York: Springer Verlag, 2007, vol. 29. 